\title{
Hiccups and Inappropriate ADH Secretion Syndrome as Presentations of Tick-Borne Disease
}

\author{
Andrea Da Porto ${ }^{1}$, Michele Battellino², Gianluca Colussi ${ }^{1}$, Vito Di Piazza ${ }^{2}$, Leonardo Sechi ${ }^{1}$ \\ ${ }^{1}$ Clinica Medica, University of Udine, Udine, Italy \\ ${ }^{2}$ Internal Medicine Department, Tolmezzo Hospital, Tolmezzo, Italy
}

Received: 20/06/2019

Accepted: 02/07/2019

Published: 24/07/2019

How to cite this article: Da Porto A, Battellino M, Colussi G, Di Piazza V, Sechi L. Hiccups and inappropriate ADH secretion syndrome as a presentations of tick-borne disease. EJCRIM 2019;6: doi:10.12890/2019_001188.

Conflicts of Interests: The Authors declare that there are no competing interest

This article is licensed under a Commons Attribution Non-Commercial 4.0 License

\section{ABSTRACT}

Tick-borne diseases (Lyme disease and tick-borne encephalitis) are becoming a major public health concern. Rapid and correct diagnosis is crucial for complicated cases but is often delayed because of low suspicion or unusual clinical presentation. In this paper the authors describe two atypical presentations of Lyme disease and tick-borne encephalitis in order to help clinicians resolve diagnostic challenges.

\section{LEARNING POINTS}

- Tick -borne diseases canould have atypical presentations.

- In endemic areas, patients with unexplained and refractory hyponatremiaaemia, should be screened for Lyme disease even in the absence of elevatedion of inflammatory markers or other specific symptoms.

- Persistent hiccups could be an atypical clinical presentation of tick-borne encephalitis (TBE).

\section{KEYWORDS}

Hyponatremia, hiccups, tick-borne encephalitis, Lyme disease

\section{INTRODUCTION}

Climate change is resulting in the rapid growth of tick populations in many European regions, with a consequent increase in the incidence of Lyme borreliosis and tick-borne encephalitis (TBE) ${ }^{[1-5]}$.

\section{CASE DESCRIPTION}

\section{Case 1}

Our patient was a 62-year-old woman with a history of gastro-resection for gastric carcinoma in situ, hepatic steatosis, gallbladder stones and mild depression. The patient lived in an Alpine region and helped her husband in his work as a forest ranger. She was admitted to our ward for a 2-week history of general weakness, confusion, dizziness and dorsal back pain. She was taking lansoprazole 15 mg/daily and acetaminophen $1-2 \mathrm{~g} /$ daily for pain relief.

On presentation, the patient was afebrile, confused, and had mild tachycardia (HR $112 \mathrm{bpm}$ ) and elevated blood pressure (160/90 mmHg). She looked dehydrated. The general physical examination (heart, lungs, abdomen) was unremarkable. Mobilization and percussion of the dorsal spine were associated with pain. Neurological examination at admission showed mild, left-sided brachial and crural muscle weakness and generalized persistent hypo-areflexia even after the Jendrassik manoeuvre. Sensation was reduced only in distal limbs. 
Laboratory findings showed a normal blood cell count, normal renal and hepatic function, glycaemia and normal thyroid function. Her CRP level was $3.43 \mathrm{mg} / \mathrm{l}$. The patient had hyponatraemia (sodium $123 \mathrm{mEq} / \mathrm{l}$ ), hypomagnesaemia (magnesium $0.67 \mathrm{mEq} / \mathrm{l}$ ) and hypokalaemia (potassium $3.0 \mathrm{mEq} / \mathrm{l})$. Uric acid was low (1.1 mg/dl). Plasma osmolarity was $246 \mathrm{mOsm} / \mathrm{kg}$ and urinary osmolarity was $466 \mathrm{mOsm} / \mathrm{kg}$. Spot urinary sodium was $118 \mathrm{mmol} / \mathrm{l}$. These findings suggested the syndrome of inappropriate antidiuretic hormone secretion (SIADH).

ECG and chest x-ray were normal. A brain CT scan was negative for ischemic or haemorrhagic lesions and encephalic masses. As a paraneoplastic syndrome (history of gastric cancer) was suspected, contrast-enhanced CT scanning was extended to the spine, thorax and abdomen without any pathological findings.

Intravenous (IV) infusion of normal saline was started in order to normalize sodium levels, with the infusion rate regulated by a validated algorithm with appropriate monitoring. However, sodium levels worsened ( $120 \mathrm{mEq} / \mathrm{l})$ with no improvement even after IV supplementation. Infusion of hypertonic saline ( $\mathrm{NaCl} 2 \%)$ was then started while blood sodium levels continued to be monitored.

Three day after admission, the patient developed left blepharospasm and left peripheral facial paralysis, with consequent speech impairment. She also experienced worsening of the initial left brachial and crural weakness such that she was unable to carry anything or bear weight. Sodium was $123 \mathrm{mEq} / \mathrm{l}$.

We performed a lumbar puncture in order to rule out acute polyneuritis. The obtained liquor was limpid, with normal glucose, high protein

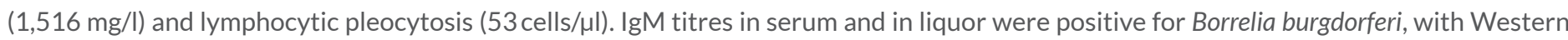
blot analysis confirming Lyme disease.

Antibiotic therapy with IV ceftriaxone $2 \mathrm{~g}$ twice a day was started according current guidelines. Sodium levels started to gradually improve 3 days after antibiotic treatment was initiated and normal levels were maintained (Fig. 1) after discontinuation of saline IV infusion. The neurological picture also improved with slow regression of the peripheral left facial deficit and progressive improvement in left brachial and crural strength. The blepharospasm also resolved.

Antibiotic treatment was continued for 21 days and the patient was discharged to a physical rehabilitation unit. The patient had completely recovered 3 months later.

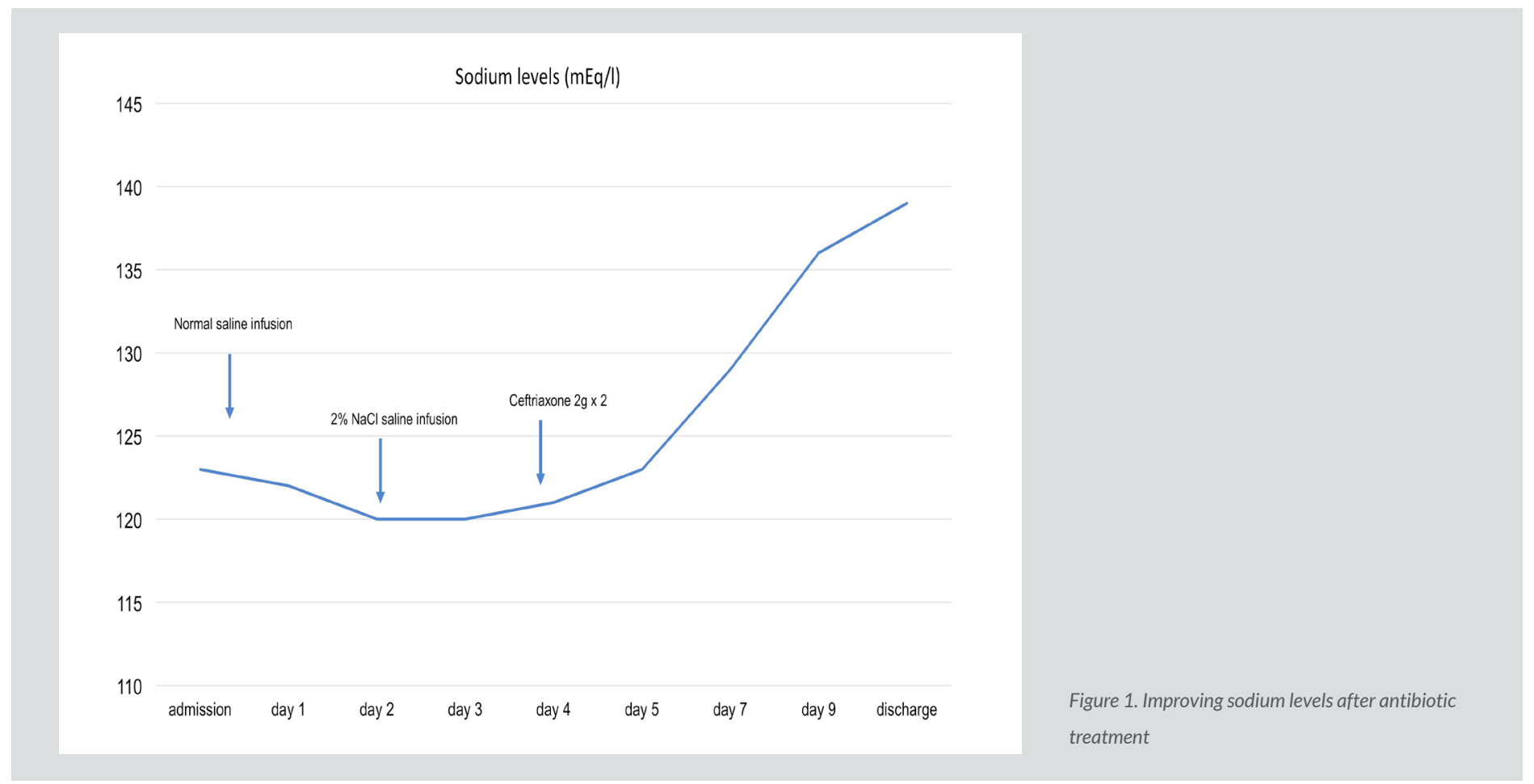

Case 2

A 52-year-old man with a history of arterial hypertension was admitted to our ward complaining of high fever and incurable hiccups which had begun 3 days previously. The patient lived in the Alpine region of Carnia and used to pick mushrooms in the woods. He had been evaluated 14 days earlier for fever and flu-like symptoms by his general practitioner who prescribed a 6-day course of antibiotic treatment 
with amoxicillin-clavulanate with temporary improvement of symptoms. The patient was taking levosulpiride $25 \mathrm{mg} / \mathrm{daily}$ for hiccups and acetaminophen 2-3 g/daily for fever relief.

On presentation, the patient had a temperature of $38.9^{\circ} \mathrm{C}$, tachycardia (HR $\left.102 \mathrm{bpm}\right)$, normal blood pressure $(112 / 80 \mathrm{mmHg}$ and continuous hiccups. The general physical examination (heart, lungs, abdomen) was unremarkable. The neurological examination at admission was negative, and no meningeal signs or cranial nerve deficits were found.

Laboratory findings showed mild leucocytosis (WBC 13,030/(mm ${ }^{3}$ ), normal haemoglobin and platelet count, normal electrolyte, renal and hepatic function, glycaemia and normal thyroid function. The CRP level was $6.93 \mathrm{mg} / \mathrm{l}$.

ECG and chest x-ray were normal. The day after admission, the patient was afebrile but still had continuous hiccups. A brain contrastenhanced CT scan was negative for ischemic or haemorrhagic lesions and encephalic tumours, but revealed two focal areas of low density with soft contrast enhancement in the right frontal basal-paramedian region and on the right apex (Fig. 2), compatible with areas of encephalic inflammation.

On further query, the patient remembered he had been bitten by a tick 35-40 days before the beginning of his symptoms.

Suspecting TBE we proceeded with a lumbar puncture. The liquor was limpid, with normal glucose, high protein and a normal cell count. We started empiric treatment for menigoencephalitis with acyclovir. Two days later, laboratory findings showed a positive lgM titre for tickborne encephalitis and negative antigens for B. burgdorferi in serum and in liquor. Hiccups were finally managed with baclofen.

Polymerase chain reaction analysis confirmed the presence of TBE antigens in liquor. The final diagnosis was tick-borne encephalitis with secondary resistant hiccups. Brain MRI conducted after resolution of symptoms was normal.

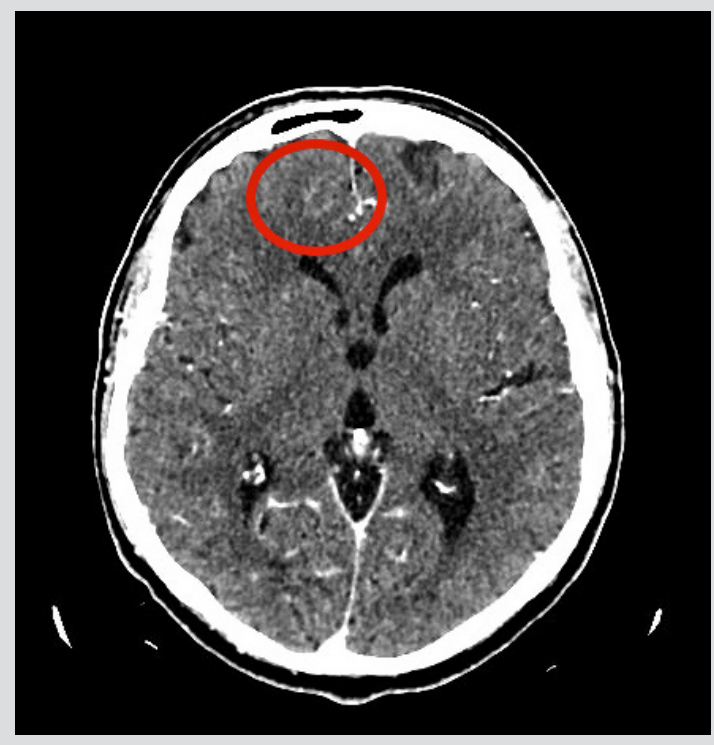

Figure 2. CT scan showing encephalic inflammation

\section{DISCUSSION}

Case 1 was unusual in that severe and refractory hyponatraemia presented at the same time as peripheral polyneuritis as the initial manifestations of Lyme disease in the absence of any other signs of infection or inflammation.

The relationship between hyponatraemia and central nervous system (CNS) disorders is well documented. Infective and inflammatory CNS conditions have also been rarely associated with SIADH and subsequent hyponatraemia. The association of hyponatraemia and neuroborreliosis is particularly rare and, as far as we know, only a few cases have been reported in the clinical literature. In 2004, Shamin et al. ${ }^{[6]}$ reported two cases of neuroborreliosis with mild hyponatraemia, neuropsychiatric symptoms, and acute demyelinating polyneuropathy with autonomic involvement. In those cases, hyponatraemia was mild and easily reversed with fluid restriction in combination with antibiotic treatment. Recently, Siddiqui et al. reported another case where hyponatraemia in an 83-year-old woman was easily managed with fluid restriction and $\mathrm{NaCl}$ oral supplements. Our patient had euvolemic hyponatraemia due to SIADH and refractory to correction with normal and hypertonic saline ( $\mathrm{NaCl} 2 \%)$ administered at a meticulous infusion rate. Normalization of sodium levels was achieved only 4 days after 
the beginning of antibiotic therapy, so we presume Borrelia infection helped maintain the hyponatraemia.

The mechanism of SIADH induced by Lyme neuroborreliosis (LNB) remains unclear. Weller and colleagues ${ }^{[7]}$ demonstrated elevated levels of the inflammatory cytokine interleukin- 6 in the serum and cerebrospinal fluid of patients with LNB, inducing antidiuretic hormone release. This evidence suggests that CNS inflammation may mediate the development and severity of SIADH. Consequently, when the underlying cause of SIADH is infective, the syndrome usually resolves only when correct antibiotic treatment is administered ${ }^{[8]}$.Case 2 is interesting because of the atypical clinical presentation and because it suggests that frontal lobe inflammation secondary to TBE can be associated with persistent hiccups. Intractable hiccups have sometimes been associated with encephalic inflammation in the literature ${ }^{[9,10]}$, although it is very rare for hiccups to be the sole presenting symptom of serious neurological disease. To our knowledge no cases of hiccups associated with TBE have been reported.

The pathophysiology of hiccups is not entirely clear, but is thought to involve a reflex arc and unilateral contraction of the diaphragm. The afferent limb of the reflex arc consists of the phrenic nerve, vagus nerve and sympathetic chain, while the efferent limb consists of the phrenic nerve with connections to the glottis and inspiratory intercostal muscles. Centrally, hiccups are thought to be mediated by a combination of the phrenic nerve nuclei, respiratory centres, the reticular formation in the brainstem, and the hypothalamus.

In our patient we found radiological signs of focal encephalic inflammation in the right frontal basal region and the ipsilateral apical frontal region, which resolved after recovery.

Irritative or destructive lesions in frontal areas mostly cause mental disorders, temporal lobe epilepsy, aphasia and paralysis. In some cases, lesions in the frontal lobes (in particular the right) have been associated with incurable hiccups. Li et al. described a case of persistent hiccups secondary to frontal lobe meningioma, while Lee reported a case due to right frontotemporal astrocytoma. In both cases, it was speculated that the intractable hiccups arose from the frontotemporal lobe, especially the frontal lobe. It was suggested that the frontotemporal area might be involved in the control mechanisms of hiccups as a stimulatory or inhibitory modulator of the reflex arc causing hiccups. We postulate that the hiccups in our patient might have been generated by abnormal neural discharges stimulated by frontal areas of inflammation which reached hiccup-mediating centres such as the brainstem and sustain hiccups.

\section{CONCLUSION}

In this paper we describe two atypical presentations of Lyme disease and TBE that could help clinicians facing diagnostic challenges. We suggest screening for Lyme disease in patients from endemic areas who have unexplained refractory hyponatraemia, even in the absence of elevated inflammatory markers or other specific symptoms. Case 2 is interesting because of the atypical clinical presentation and because it confirms that frontal lobe inflammation secondary to TBE may be associated with persistent hiccups.

\section{REFERENCES}

1. Sprong H, Hofhuis A, Gassner F, Takken W, Jacobs F, van Vliet AJ. Circumstantial evidence for an increase in the total number and activity of Borrelia-infected Ixodes ricinus in the Netherlands. Parasit Vectors 2012;5(1):294.

2. Dubrey SW, Bhatia A, Woodham S, Rakowicz W. Lyme disease in the United Kingdom. Postgrad Med J 2014;90(1059):33-42.

3. Trájer A, Bobvos J, Páldy A, Krisztalovics K. Association between incidence of Lyme disease and spring-early summer season temperature changes in Hungary--1998-2010. Ann Agric Environ Med 2013;20(2):245-251.

4. Septfons A, Goronflot T, Jaulhac B, Roussel V, De Martino S, Guerreiro S, et al. Epidemiology of Lyme borreliosis through two surveillance systems: the national Sentinelles GP network and the national hospital discharge database, France, 2005 to 2016. Euro Surveill 2019;24(11).

5. Hellenbrand W, Kreusch T, Böhmer MM, Wagner-Wiening C, Dobler G, Wichmann O, et al. Epidemiology of tick-borne encephalitis (TBE) in Germany, 2001-2018. Pathogens 2019;8:42.

6. Shamin EA, Shamin SA, Liss G, Nylen E, Pincus JH, Yepes M. Constipation heralding neuroborreliosis: an atypical tale of 2 patients. Arch Neurol 2005;62:671-673.

7. Weller M, Stevens A, Sommer N, Wiethölter H, Dichgans J. Cerebrospinal fluid interleukins, immunoglobulins, and fibronectin in neuroborreliosis. Arch Neurol 1991;48:837841.

8. Perkins MP, Shumway N, Jackson WL Jr. Lyme neuroborreliosis presenting as the syndrome of inappropriate antidiuretic hormone secretion. MedGenMed 2006;8(3):71.

9. Sugimoto T, Takeda N, Yamakawa I, Kawai H, Tanaka Y, Sakaguchi M, et al. Intractable hiccup associated with aseptic meningitis in a patient with systemic lupus erythematosus. Lupus 2008;17(2):152-153.

10. Hemachudha T, Phanthumchinda K, Indrakoses A, Wilde HG. Intractable hiccups (singultus) as presenting manifestation of Japanese encephalitis. J Med Assoc Thai 1984;67(11):621-623 